\title{
A robust probabilistic evaluation of soil-foundation-structure interaction effects on structural response
}

\author{
M. Moghaddasi K., M. Cubrinovski, S. Pampanin \& A. Carr \\ Civil and Natural Resources Engineering, University of Canterbury, New Zealand \\ J.G. Chase \\ Mechanical Engineering Department, University of Canterbury, New Zealand
}

\begin{abstract}
Evaluation of soil-foundation-structure interaction (SFSI) effects on structural response is a challenging task because of the involved impact of uncertainties in system's parameters and randomness in the input earthquake motion. In this paper, a rational method is utilized to elucidate these effects through the use of a rheological soil-foundation-structure model in a robust probabilistic simulation. Realistic, but random models with varied soil and structural parameters within relevant range of values are generated out of a Monte Carlo simulation and subjected to an ensemble of earthquake motions. Both linear and nonlinear structural behavior due to earthquake motion is considered. The SFSI effects on linear structural response are quantified by the median response and the accompanied dispersion and are then used as a reference when evaluating the SFSI effects for superstructures with nonlinear behavior. In addition, for the structural systems with linear behavior detrimental SFSI scenarios are indentified. The achieved quantification of SFSI effects can be used as a significant step towards a reliable seismic design framework incorporating SFSI. Note that in all analyses the nonlinearity in soil behavior has been accounted for through the equivalent linear approach.
\end{abstract}

\section{INTRODUCTION}

A robust evaluation of soil-foundation-structure interaction (SFSI) effects on structural response with a consistent outcome needs to consider the combined impact of the uncertainty in soil and structural parameters along with the inherent randomness of the input ground motion. Without respecting this fact and based on the traditional quantification of SFSI effects via period lengthening and damping increase (Jennings \& Beilak 1973, Veletsos \& Meek 1974, Veletsos \& Nair 1975), it has been concluded and implemented in major design codes (ATC-3-06 1984, FEMA 440 2005) that SFSI consideration in the dynamics analysis is beneficial. However, it has been also recognized that soil-structure interaction effects may be detrimental (Gazetas \& Mylonakis 1998, Mylonakis \& Gazetas 2000, Dutta \& Bhattacharya 2004) and increase the structural response as compared to a fixed base model.

To investigate the above mentioned SFSI effects on the structural response, a probabilistic method was utilized, an approach which is gaining a growing attention in geotechnical engineering community, especially in SFSI studies (Jin \& Lutes 2000, Lutes et al. 2000). In this study, the effects of soil and structural variability have been investigated in conjunction with the randomness of the input earthquake motion. An idealized, but commonly accepted soil-shallow foundation-structure (SSFS) model was adopted for the analysis representing the superstructure as a SDOF system and the soil-foundation element as an equivalent linear cone model with frequency independent coefficients (Wolf 1994, Stewart 2003). Two series of analyses were conducted in which the superstructure was modeled as a linear or nonlinear system respectively in order to investigate the effect of structural nonlinearity in the SFSI problem. A Monte Carlo simulation was utilized to generate all the random parameters of the analytical model in such a way that all generated models represented realistic soil-foundationstructure systems. The generated SSFS models were then subjected to a suite of 40 earthquake motions recorded on stiff/soft soils to account for variability in the input motion characteristics. Hence, soil, structural and earthquake motion variability were systematically covered in the analyses.

In this paper, the effects of SFSI on the linear structural response were investigated by using the median response and associated dispersion to build up a conceptual understanding of the SFSI effects and evaluate the prevailing engineering view implemented in the seismic design codes. Following this quantification, cases with detrimental effects were scrutinized in order to identify a relation between characteristics of the SFSI system and earthquake causing increased structural response when incorporating SFSI effects. Finally, the influence of 
structural nonlinearity in the SFSI problem was evaluated by comparing the median structural responses in linear and nonlinear structural systems.

\section{PROBABILISTIC SIMULATIONS}

\subsection{Methodology}

To elucidate the effects of SFSI on the structural response, a probabilistic simulation using a wide range of SSFS systems subjected to a range of earthquake motions with different characteristics were considered. A fairly simple rheological SSFS model (defined in Sec. 2.2) was implemented in the analyses. Its parameters were systematically defined randomly through a Monte Carlo simulation by carefully ensuring to satisfy the requirements of realistic SSFS models and nonlinear soil behavior (explained in Sec. 2.3). The generated SSFS models were then imposed to an ensemble of 40 earthquake ground motions recorded on stiff/soft soils to account for variability in the input motion spectrum and type (introduced in Sec. 2.4). A closed-form solution programmed in MATLAB was used for the analyses with linear structures whereas the analyses with nonlinear superstructures were conducted using an FEM code (Ruaumoko 2D). Results from the analysis were illustrated through a comprehensive statistical presentation, described in Section 2.5.

\subsection{Soil-shallow foundation-structure model}

The adopted SSFS model was constructed by a single-degree-of-freedom (SDOF) superstructure linked to a lumped-parameter soil-foundation element by a rigid beam as shown in Figure 1. The superstructure is characterized by: (i) structural mass participating in the fundamental mode of vibration, $\mathrm{m}_{\text {str }}$, (ii) initial structural lateral stiffness, $\mathrm{k}_{\mathrm{str}}$, (iii) $5 \%$ equivalent viscous damping, $\xi$, and (iv) effective height considered from the foundation level to the centre of the inertial loads, $\mathrm{h}_{\mathrm{eff}}$.

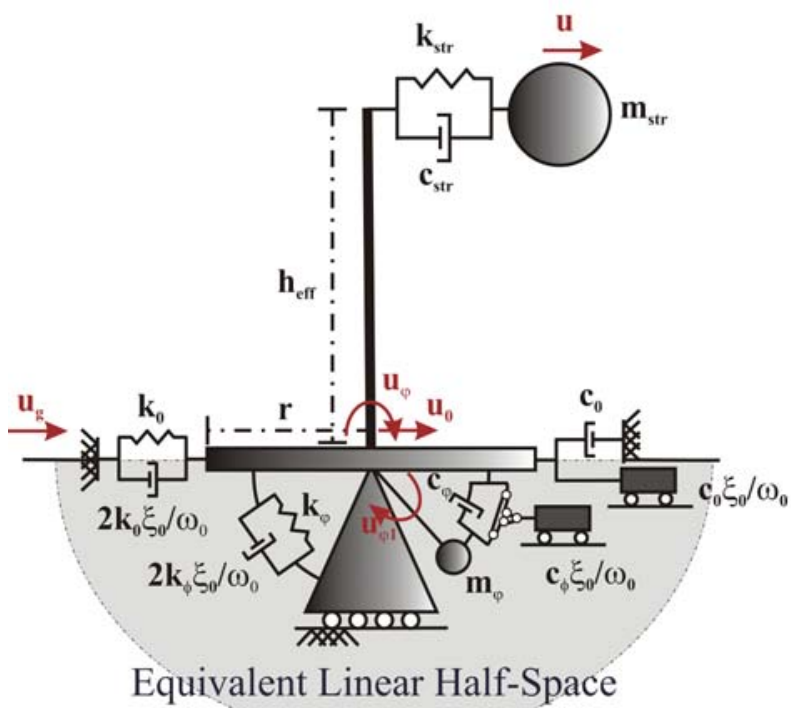

Figure 1. Coupled dynamic soil-shallow foundation-structure model for horizontal and rocking foundation motions.
The soil-foundation element was modeled by a discrete model representing a shallow foundation resting on a linear elastic half space (Wolf 1994) with the frequency independent coefficients. The parameters of the soil-foundation element are evaluated using the following formulae:

1 Horizontal stiffness and damping:

$$
\mathrm{k}_{0}=\frac{8 \mathrm{Gr}}{2-v}, \mathrm{c}_{0}=\rho \mathrm{V}_{\mathrm{s}} \mathrm{A}
$$

2 Rocking stiffness:

$$
\mathrm{k}_{\varphi}=\frac{8 \mathrm{Gr}^{3}}{3(1-v)}
$$

3 Rocking damping:

$$
\begin{aligned}
& \text { if } v \leq 1 / 3: c_{\varphi}=\rho V_{p} I_{r} \\
& \text { if } 1 / 3 \leq v \leq 1 / 2: c_{\varphi}=\rho\left(2 V_{s}\right) I_{r}
\end{aligned}
$$

4 Rocking added mass:

if $1 / 3 \leq v \leq 1 / 2: \Delta \mathrm{m}_{\varphi}=1.2(v-1 / 3) \rho \mathrm{I}_{\mathrm{r}} \mathrm{r}$

5 Rocking internal mass moment of inertia:

$$
\begin{aligned}
& \text { if } v \leq 1 / 3: \mathrm{m}_{\varphi}=\frac{9 \pi}{32} \rho \mathrm{I}_{\mathrm{r}} \mathrm{r}(1-v)\left(\frac{\mathrm{V}_{\mathrm{p}}}{\mathrm{V}_{\mathrm{s}}}\right)^{2} \\
& \text { if } 1 / 3 \leq v \leq 1 / 2: \mathrm{m}_{\varphi}=\frac{9 \pi}{8} \rho \mathrm{I}_{\mathrm{r}} \mathrm{r}(1-v)
\end{aligned}
$$

where $r$ is the equivalent radius of the foundation, $A$ is the area of the foundation $\left(A=\pi r^{2}\right), I_{r}$ is the mass moment of inertia for rocking motion $\left(\mathrm{I}_{\mathrm{r}}=\pi \mathrm{r}^{4} / 4\right)$, and $\rho, v, V_{s}, V_{p}$ are soil mass density, Poisson's ratio, soil shear wave velocity and longitudinal wave velocity, respectively.

Structural nonlinearity due to earthquake motion was considered through two types of hysteretic model: Takeda degrading stiffness (TK) and bilinear elasto-plastic (EP) representing the concrete framed and steel framed structures, respectively. To incorporate soil nonlinearity into the adopted soilfoundation element, the conventional equivalent linear method was utilized (Seed 1970). This approach is based on representing the soil nonlinearity by choosing the reduced soil characteristics which are compatible with the free field strains and induced by the propagating seismic waves.

\subsection{Generation of models with random parameters}

To investigate the SFSI effects on structural response a design spectrum style was selected. In this spectrum, a period range of $0.2,0.3 \ldots 1.8(\mathrm{sec})$ was defined to: (i) represent the fixed-base (FB) superstructures with 3-30 (m) height and (ii) satisfy the period-height relationship introduced in NZS1170.5. For each considered period $\left(\mathrm{T}_{\mathrm{FB}}\right), 1000$ SSFS models were generated via assembling the randomly de- 
fined parameters for the soil-foundationsuperstructure system. These parameters are defined either by random selection from a defined range or by using predefined parameters in a commonly accepted relationship. The number 1000 was chosen with the intention to: (i) give the best fit uniform distribution for the randomly selected parameters and (ii) increase the accuracy of the Monte-Carlo simulation compared to the exact expected solution (Fishman 1995). The procedure adopted in defining the parameters is elaborated below:

1. Initial soil shear wave velocity $\left[\mathrm{V}_{\mathrm{s}}\right]$ was selected in the range between 80 and $360 \mathrm{~m} / \mathrm{sec}$ representing soft to relatively stiff soils.

2. Shear wave velocity degradation ratio $\left[\left(\mathrm{V}_{\mathrm{s}}\right)_{\mathrm{sec}} /\left(\mathrm{V}_{\mathrm{s}}\right)_{0}\right]$ was selected from the variation range of 0.15-0.7 assuming that the induced shear strain in the soil due to the earthquake motion was in the range of $0.01-1 \%$. This range was selected as a representative ground response considering the fact that the employed earthquake motions have magnitudes between 6.2 and 7.6, and source-to-site distances of less than $40 \mathrm{~km}$.

3 . Soil mass density $[\rho]$ was selected from the variation range of $1600-1900 \mathrm{~kg} / \mathrm{m}^{3}$.

4. Poisson's ratio [v] was selected from the variation range of 0.3-0.45.

5. Degraded shear modulus $\left[\mathrm{G}_{\mathrm{sec}}\right]$ was defined through the previously introduced degraded shear wave velocity, $\left(\mathrm{V}_{\mathrm{s}}\right)_{\mathrm{sec}}$ as:

$\mathrm{G}_{\mathrm{sec}}=\rho\left(\mathrm{V}_{\mathrm{s}}\right)_{\mathrm{sec}}^{2}$

6. Soil material damping $\left[\xi_{0}\right]$ was defined at the effective circular frequency of SFSI system $\left(\omega_{0}\right)$ by implementing $\left(\mathrm{V}_{\mathrm{s}}\right)_{\mathrm{sed}}\left(\mathrm{V}_{\mathrm{s}}\right)_{0}$ ratio in the following expression:

$\frac{25-\xi_{0}}{25-10}=\frac{\left(\mathrm{V}_{\mathrm{s}}\right)_{\mathrm{sec}} /\left(\mathrm{V}_{\mathrm{s}}\right)-0.15}{0.7-0.15}$

Equation 7 represents the linear variation of damping between 10\%-25\% corresponding to the velocity degradation ratio of $0.7-0.15$.

7. Effective height of the superstructure $\left[h_{\text {eff }}\right]$ was selected from the variation ranges defined in Table 2. These limitations are based on: (i) a typical period-height relationship adopted in NZS 1170.5 and (ii) the considered limitation for the structural total height, $3-30 \mathrm{~m}$.

Table 1. Ranges of variation for $\mathrm{h}$

\begin{tabular}{cc}
\hline $\mathrm{T}_{\mathrm{FB}}(\mathrm{sec})$ & $\mathrm{h}(\mathrm{m})$ \\
\hline $0.2-0.32$ & $2-26.8 \mathrm{~T}_{\mathrm{FB}}^{1.33}$ \\
$0.32-0.8$ & $9.1 \mathrm{~T}_{\mathrm{FB}}^{1.33}-26.8 \mathrm{~T}_{\mathrm{FB}}^{1.33}$ \\
$0.8-1.8$ & $9.1 \mathrm{~T}_{\mathrm{FB}}^{1.33}-20$ \\
\hline
\end{tabular}

8. Foundation radius [r] was selected from variation range defined in Table 3. To define these limitations, the building aspect ratio $\left(\mathrm{h}_{\mathrm{eff}} / \mathrm{r}\right)$ was used.
It was assumed that $h_{\text {eff }} / \mathrm{r}$ ratio for conventional building structures varies in between 1 and 4 and also $r$ is limited to the range of $2-12 \mathrm{~m}$, representing structures having 1-3 bays with length of 4-8 m each.

Table 2. Ranges of variation for $r$

\begin{tabular}{cc}
\hline $\mathrm{h}(\mathrm{m})$ & $\mathrm{r}(\mathrm{m})$ \\
\hline $2-8$ & $2-\mathrm{h}$ \\
$8-12$ & $\mathrm{~h} / 4-\mathrm{h}$ \\
$12-20$ & $\mathrm{~h} / 4-12$ \\
\hline
\end{tabular}

9. Structural mass $\left[\mathrm{m}_{\text {str }}\right]$ was defined based on relative mass index $(\overline{\mathrm{m}})$, which is defined as:

$\overline{\mathrm{m}}=\frac{\mathrm{m}_{\mathrm{str}}}{\rho \mathrm{r}^{2} \mathrm{~h}}$

A uniform distribution for $\overline{\mathrm{m}}$ within the range of 0.4-0.6 representing conventional building structures (Stewart 1999) was considered and the predefined values for $\rho, r$ and $h$ were implemented in Equation 8 to define $\tilde{\mathrm{m}}$.

10. Initial structural lateral stiffness $\left[\mathrm{k}_{\text {str }}\right]$ : was defined by implementing the predefined values of $\mathrm{m}_{\text {str }}$ in:

$\mathrm{k}_{\mathrm{str}}=\frac{4 \pi^{2}}{\mathrm{~T}_{\mathrm{FB}}^{2}} \mathrm{~m}_{\mathrm{str}}$

11. Structural damping coefficient $\left[\mathrm{c}_{\mathrm{str}}\right]$ was defined by implementing the predefined values for $m_{\text {str }}$ and $\mathrm{k}_{\mathrm{str}}$ in:

$\mathrm{c}_{\mathrm{str}}=2(0.05) \sqrt{\mathrm{k}_{\mathrm{str}} \mathrm{m}_{\mathrm{str}}}$

12. Predominant period of the SSFS system $\left[\mathrm{T}_{\mathrm{SSFS}}\right]$ was defined as:

$\mathrm{T}_{\mathrm{SSFS}}=\mathrm{T}_{\mathrm{FB}} \sqrt{1+\frac{\mathrm{k}_{\mathrm{str}}}{\mathrm{k}_{0}}+\frac{\mathrm{k}_{\mathrm{str}} \mathrm{h}^{2}}{\mathrm{k}_{\varphi}}}$
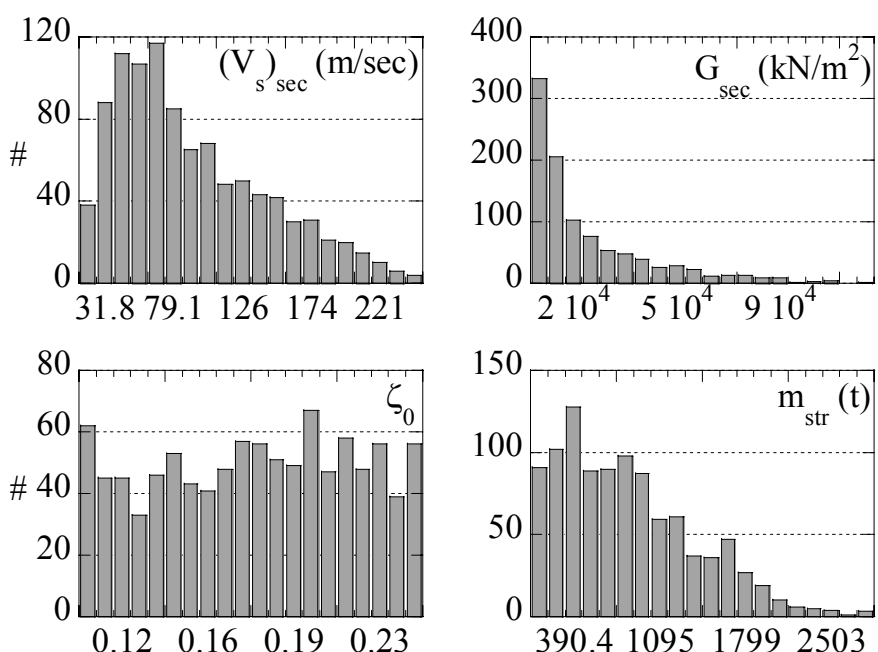

Figure 2. Distributions of randomly generated models for $\mathrm{T}_{\mathrm{FB}}=1.0$ sec: (a) degraded shear wave velocity, (b) degraded shear modulus (c) soil material damping and (d) structural mass 
As an example of randomly generated distributions used in the analyses, Figure 2 illustrates the distribution of $\left(V_{\mathrm{s}}\right)_{\mathrm{sec}}, \mathrm{G}_{\mathrm{sec}}, \xi_{0}$ and $\mathrm{m}_{\mathrm{str}}$ for a superstructure with a fixed base period of $\mathrm{T}_{\mathrm{FB}}=1.0(\mathrm{sec})$.

\subsection{Selection of input earthquake motions}

A suite of 40 earthquake motions recorded on stiff/soft soil (specifically, type C and D based on USGS classification) was used in the adopted timehistory simulations. The number 40 was chosen to obtain an estimate of median response within a factor \pm 0.1 with $95 \%$ confidence (Shome et al. 1998). The records were constrained as follows: (i) the magnitude in the range of 6.5-7.5, (ii) the closest distance to the fault rupture in the range of $15-40 \mathrm{~km}$ and (iii) the peak ground acceleration (PGA) greater than $0.1 \mathrm{~g}$.

The selected records were then scaled to have reasonably distributed PGAs within the range of 0.3$0.8 \mathrm{~g}$, assuming that a nonlinear behavior of the superstructure will be caused by those levels of intensity. Respecting rigorous scaling criteria and recommendations in NZS 1170.5, all scaling factors were chosen to be less than 3.0.

\subsection{Presentation of results from the analyses}

Two aspects of structural response were considered for this investigation: (i) structural distortion $(\mathrm{u})$ and (ii) structural total displacement, $\left(\mathrm{u}_{\text {str }}\right)$. Structural distortion is the horizontal displacement of the superstructure relative to the foundation that represents the transmitted displacement/force to the superstructure. The structural total displacement is defined as the summation of the horizontal foundation displacement, structural lateral displacement due to foundation rocking and structural distortion, and it represents the expected top floor displacement.

In order to simplify the presentation of the results from numerous time-history simulations, only the maximum values of the selected structural response parameters were considered. These values are presented in a normalized format as a ratio with respect to the results obtained from corresponding fixed base (FB) systems when subjected to the same earthquake excitation. Based on this type of presentation, SFSI is recognized to be detrimental when: (i) structural distortion modification factor $\left(\mathrm{u}_{\mathrm{SSFS}} / \mathrm{u}_{\mathrm{FB}}\right)$ is greater than unity; or (ii) structural total displacement modification factor $\left(\left(\mathrm{u}_{\mathrm{str}}\right)_{\mathrm{SSFS}} /\left(\mathrm{u}_{\mathrm{str}}\right)_{\mathrm{FB}}\right)$ is greater than unity.

The resulted normalized values are presented in a box and whisker plot format to characterize the central tendency (median) and the accompanied dispersion at different levels of probability. In a box and whisker plot, the box has lines at $25^{\text {th }}$ percentile $\left(1^{\text {st }}\right.$ quartile), median, and $75^{\text {th }}$ percentile ( $3^{\text {rd }}$ quartile) values. Whiskers extend from each end of the box to the $5^{\text {th }}$ percentile and $95^{\text {th }}$ percentile respectively.
Outliers are the data with values beyond those indicated by the whiskers.

\section{RESULTS AND DISCUSSION}

\subsection{SFSI effects on linear structures}

Figure 3 illustrates the results for $\mathrm{u}_{\mathrm{SSFS}} / \mathrm{u}_{\mathrm{FB}}$ (structural distortion modification factor) for all groups of SSFS models categorized based on $\mathrm{T}_{\mathrm{FB}}$. Clearly, consideration of SFSI may cause structural distortion to be increased in some cases even up to 1.8 times, however, the median of the $\mathrm{u}_{\mathrm{SSFS}} / \mathrm{u}_{\mathrm{FB}}$ ratio is less than unity through all period ranges. The evaluated risk of having detrimental effects in terms of structural distortion is in the order of $20-30 \%$ for stiff structures $\left(\mathrm{T}_{\mathrm{FB}}<0.5 \mathrm{sec}\right)$ and $10-15 \%$ for more flexible structures, as shown in Figure 3.

The impact of SFSI consideration on the structural total displacement is shown in Figure 4 by presenting $\left(\mathrm{u}_{\mathrm{str}}\right)_{\mathrm{SSFS}} /\left(\mathrm{u}_{\mathrm{str}}\right)_{\mathrm{FB}}$ (structural total displacement modification factor). Noticeably, foundation flexibility may cause structural total displacement to increase as much as 15 times, however, in $50 \%$ or more of all cases, it does not cause a significant change in the response. The risk of detrimental effects in this case of structural total displacement is in the order of $50-80 \%$ for stiff structures $\left(\mathrm{T}_{\mathrm{FB}}<0.5 \mathrm{sec}\right)$ and $40-50 \%$ for more flexible structures.

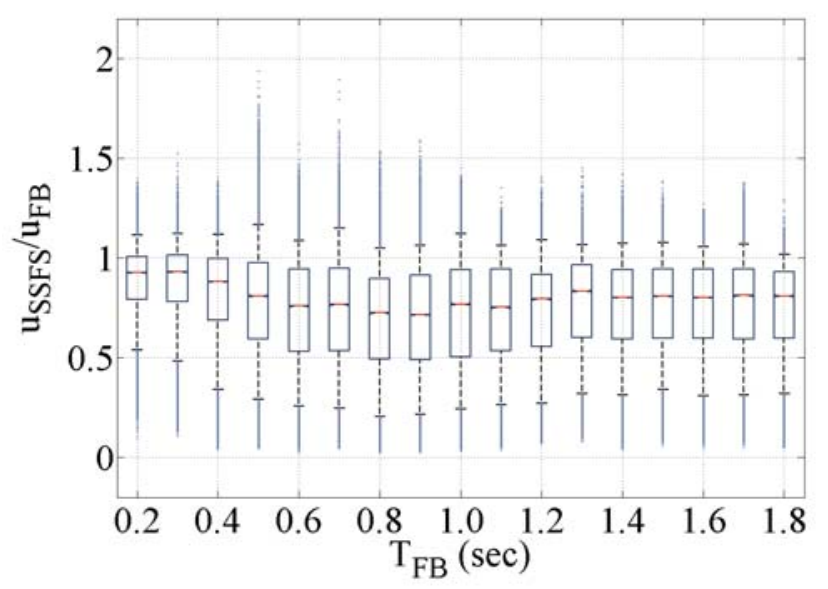

Figure 3. Structural distortion modification spectrum

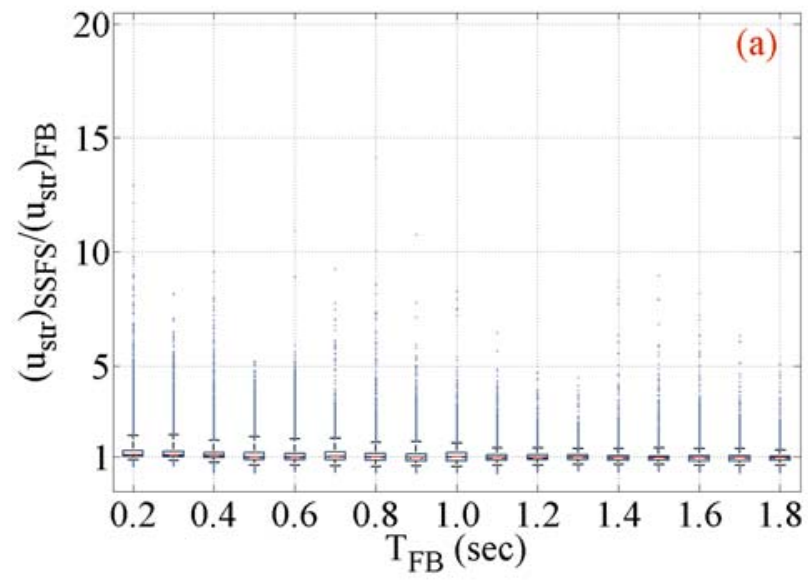




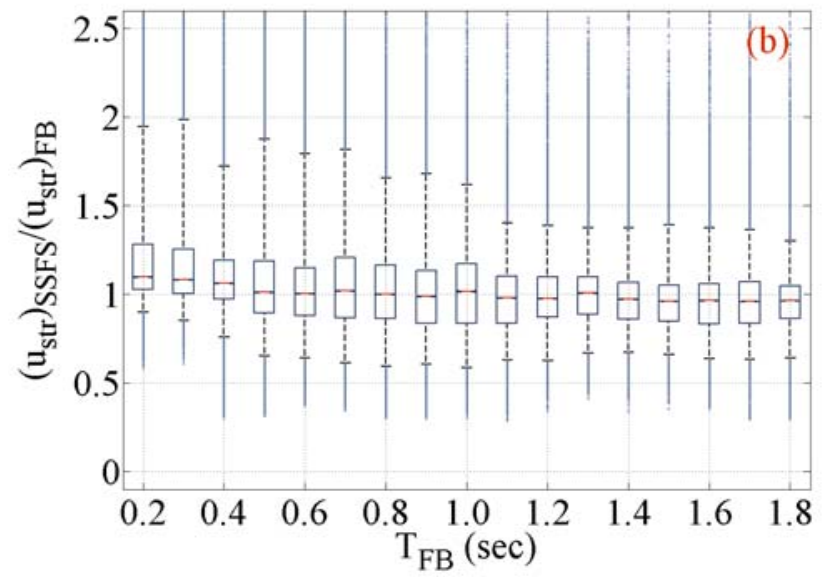

Figure 4. Structural total displacement modification spectrum in: (a) global view, (b) close view around unit.

\subsection{Identification of detrimental SFSI scenarios in terms of structural strength demand}

Since it is recognized that SFSI consideration can cause an increase in the structural distortion (or strength demand in linear analysis), contradicting to the prevailing view in most conventional building design codes (ATC-3-06 1984, FEMA 440 2005), it is important to identify scenarios for which a consideration of SFSI effects will cause an increase in the structural response. As already perceived, it is the combined effect of SSFS system properties along with the characteristics of the earthquake motion that may result in detrimental SFSI effects on structural responses. This fact is demonstrated in Figure 5 , as an example, by the histogram of earthquake motions causing an amplification in structural distortion for the set of models with $\mathrm{T}_{\mathrm{FB}}=1.0$. Clearly, for some earthquake motions the SFSI effects increased the structural response for many soil-foundationstructure systems while for other earthquakes the SFSI effects were either trivial or absent.

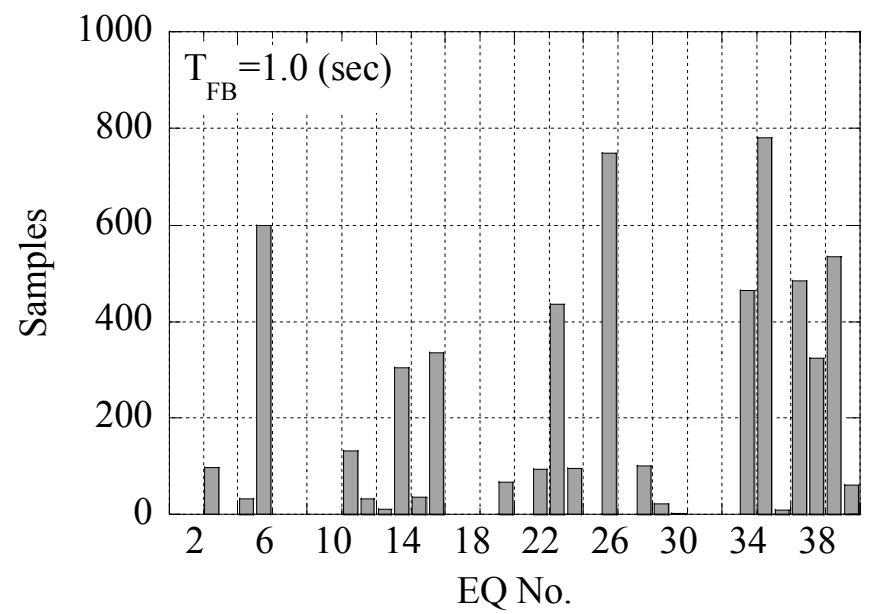

Figure 5. Histogram of the earthquake motions causing $\mathrm{u}_{\mathrm{SSFS}} / \mathrm{u}_{\mathrm{FB}}>1.0$ for group of models with $\mathrm{T}_{\mathrm{FB}}=1.0(\mathrm{sec})$

In order to investigate what characteristic of the motion makes an earthquake to produce an amplification in the structural response, the maximum acceleration response of the SSFS models are com- pared with the maximum acceleration response of the FB models (acceleration response spectrum) for two types of earthquakes: one with significant detrimental effects (increase in the structural response due to SFSI effects) and the other with no detrimental effects. Figure 6 shows this comparison for models with $\mathrm{T}_{\mathrm{FB}}=1.0 \mathrm{sec}$ and for earthquakes number 23 and 2, where $\left(\mathrm{S}_{\mathrm{a}}\right)_{\mathrm{EQ}},\left(\mathrm{a}_{\mathrm{t}}\right)_{\mathrm{FB}}$ and $\left(\mathrm{a}_{\mathrm{t}}\right)_{\mathrm{SSFS}}$ are the earthquake acceleration response spectrum, acceleration response for the FB model and the corresponding SSFS models respectively. As illustrated in this figure, the response of SSFS models (points represented by hollow circle) nearly follows the acceleration response spectrum of the earthquake (solid line), however, it could be either beyond or bellow the spectrum line. The key difference between the two spectra presented in Figures $6 \mathrm{a}$ and $6 \mathrm{~b}$ is that for periods slightly greater than $\mathrm{T}_{\mathrm{FB}}$ they show an ascending or descending branch in the spectrum respectively.
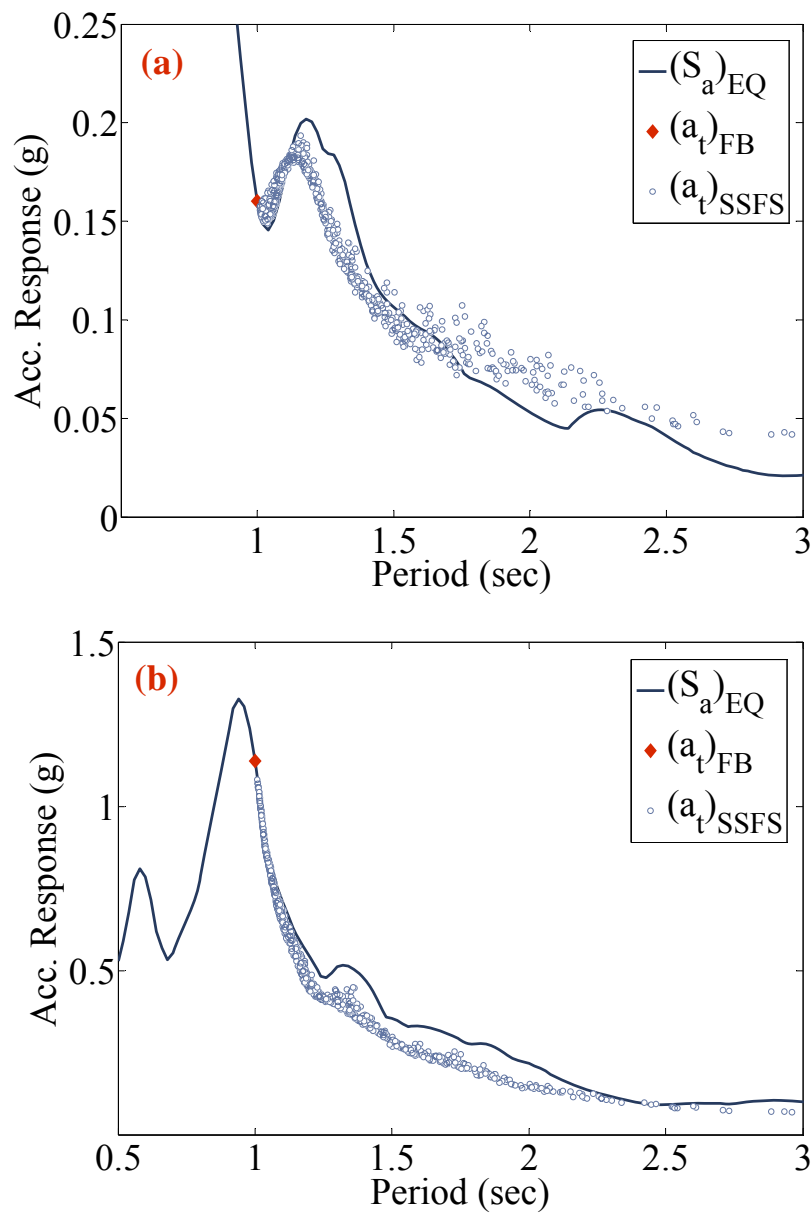

Figure 6. Comparison between the earthquake acceleration response spectrum and the acceleration response of the SSFS systems for: (a) EQ 23 and (b) EQ 2 at $\mathrm{T}_{\mathrm{FB}}=1.0$ (sec)

The observed behavior could be conceptually summarized as depicted in Figure 7. The figure indicates that in order to define whether SFSI consideration is beneficial or detrimental, the response of two systems: (i) the original $\mathrm{FB}$ system and (ii) the equivalent FB counterpart of the original SSFS system needs to be compared using the acceleration spectrum of the earthquake input motion. The equiv- 
alent FB system is defined as a FB system with the same mass and stiffness as the original SSFS system and with a modification in the structural damping. This equivalent system is also subjected to a modified input earthquake motion. The period of the SSFS system (which is also equals to the period of the equivalent fixed-base system) is always greater than the period of the original fixed-base system $\left(\mathrm{T}_{\mathrm{SSFS}}>\mathrm{T}_{\mathrm{FB}}\right)$, and hence, due to this period shift, the response of the original $\mathrm{FB}$ system, $\mathrm{S}_{\mathrm{a}}\left(\mathrm{T}_{\mathrm{FB}}\right)$, is shifted to $\mathrm{Sa}\left(\mathrm{T}_{\mathrm{SSFS}}\right)$ on the related earthquake spectrum. In addition, as a result of the modification in damping of the superstructure and the imposed input earthquake, the actual response of the equivalent FB system, $\left(a_{t}\right)_{S S F S}$, varies either beyond or bellow $\mathrm{S}_{\mathrm{a}}\left(\mathrm{T}_{\mathrm{FB}}\right)$. Based on this reasoning, if the resulting $\left(a_{t}\right)_{S S F S}$ is greater than $\mathrm{S}_{\mathrm{a}}\left(\mathrm{T}_{\mathrm{FB}}\right)$, then detrimental SSFS effects should be expected. Clearly, depending on the earthquake spectrum characteristics in the region of the fundamental periods of the fixed-base system and respective SSFS system, SFSI may results either in detrimental or beneficial effects.

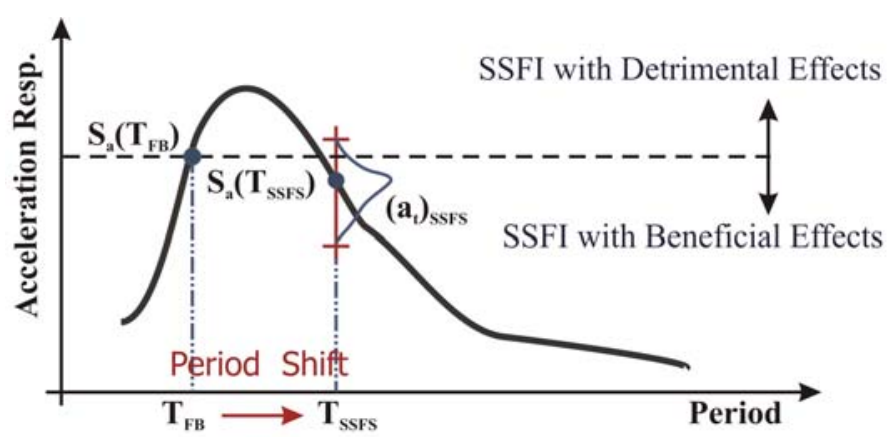

Figure 7. Conceptual presentation of SFSI detrimental/beneficial effects

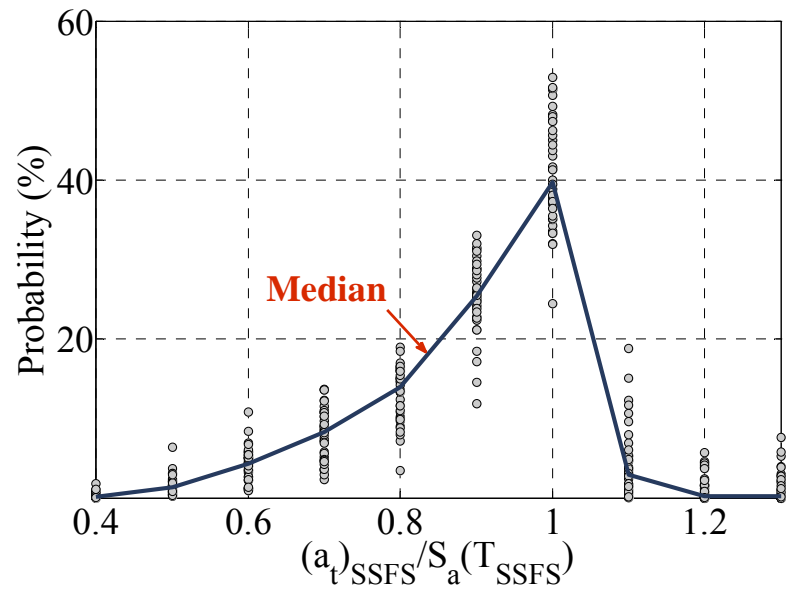

Figure 8. Probability of $\left(\mathrm{a}_{\mathrm{t}}\right)_{\mathrm{SSFS}} / \mathrm{S}_{\mathrm{a}}\left(\mathrm{T}_{\mathrm{SSFS}}\right)$ ratio for all EQs and models

In order to quantify the variation of $\left(a_{t}\right)_{S S F S} / S_{a}\left(T_{F B}\right)$, its probability of occurrence through the related variation range was evaluated. Figure 8 illustrates this quantification. In this figure, each circle represents the probability of a certain value of $\left(a_{t}\right)_{S S F S} / S_{a}\left(T_{F B}\right)$ among all the resulted values of $\left(a_{t}\right)_{S S F S} / S_{a}\left(T_{F B}\right)$ for an earthquake motion and all models. In addition, Figure 8 shows the median probability curve which is produced to represent the likelihood of $\left(a_{t}\right)_{S S F S} / S_{a}\left(T_{F B}\right)$ for $50 \%$ of the cases and more. Clearly, $\left(a_{t}\right)_{S S F S} / S_{a}\left(T_{F B}\right)$ ratio varies in between $0.4-1.3$ and it is more probable to vary in the range of $0.8-1$.

\subsection{Effects of structural nonlinearity}

To investigate the influence of structural nonlinearity on the SFSI effects, Figures 9 and 10 compares the median values of the modification in structural distortion and structural total displacement between all the three considered structural systems (linear, TK and EP), respectively,

As illustrated in Figure 9, for 50\% of the cases and more, consideration of SFSI in nonlinear structural systems causes a reduction in the expected deformation of the superstructure similar to what is observed in linear cases. However, the expected reduction factor decreases due to nonlinearity in structural behavior. Thus, it can be concluded that SFSI effect on structural distortion is more critical when structural nonlinearity is expected.

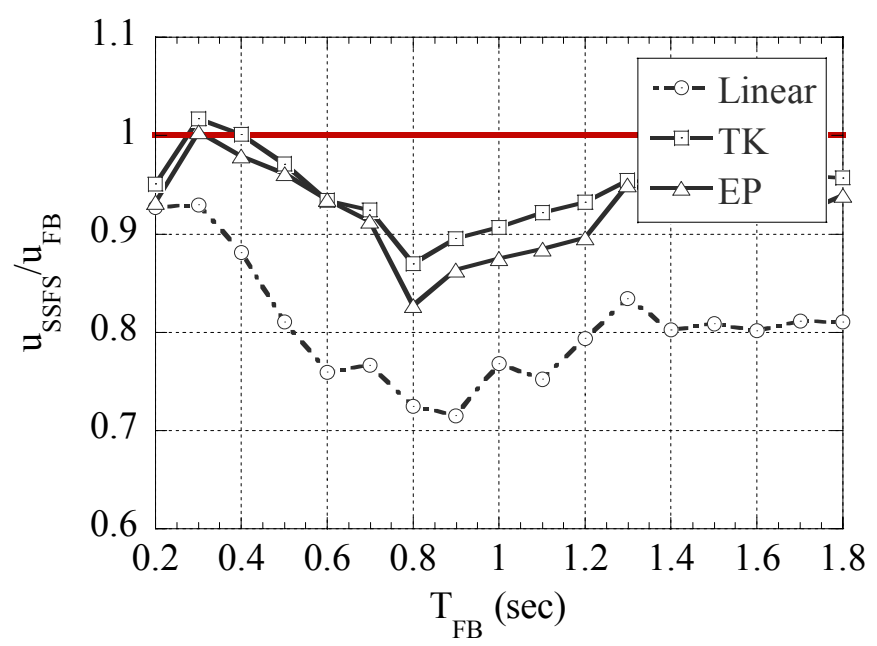

Figure 9. Comparison between median structural distortions for linear, TK, EP structural systems

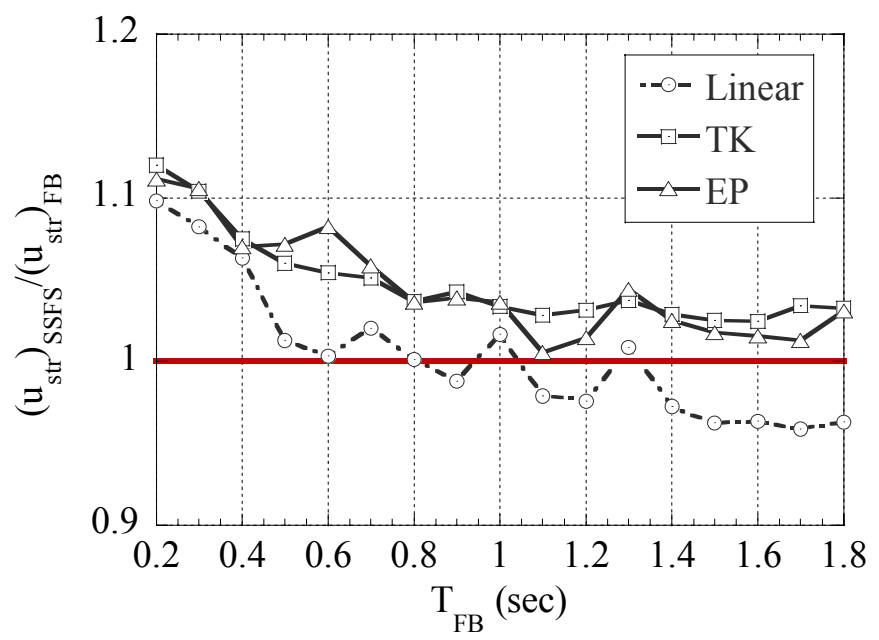

Figure 10. Comparison between median structural total displacement for linear, TK, EP structural systems 
In terms of structural total displacement, as shown in Figure 10, for the $50^{\text {th }}$ percentile response, an amplification ratio up to 1.1 is observed for nonlinear structural systems. Noted that for linear structural systems, except for stiff structures $\left(\mathrm{T}_{\mathrm{FB}}<0.5\right.$ $\mathrm{sec}$ ), consideration of SFSI does not cause a significant change in the response. Thus, structural nonlinearity amplifies SFSI effect on structural total displacement.

\section{CONCLUSION}

Soil-foundation-structure interaction (SFSI) effects on structural response have been investigated through a robust probabilistic study covering uncertainties in model parameters and input earthquake motion. In the numerical simulations, an established rheological soil-shallow foundation-structure (SSFS) model representing: (i) a single-degree-of-freedom (SDOF) superstructure with linear and nonlinear hysteretic behavior and (ii) a shallow foundation resting on an equivalent linear half-space was utilized. The parameters of the model were randomly generated via a Monte Carlo simulation in a way to result in realistic SSFS systems. All the adopted models were subjected to an ensemble of 40 earthquake motions. Key findings from the analyses can be summarized as follows:

1. Consideration of SFSI in dynamic analysis with linear structural behavior may increase the structural distortion up to 2 times, even though, in a median sense, a reduction is expected. The risk of having an increase in the structural distortion is on the order of $10-30 \%$ depending on the stiffness of the superstructure. For the total structural horizontal displacement this value is on the order of $40-80 \%$.

2. Detrimental SFSI effects or increase in the structural distortion occur for a specific earthquake spectrum characteristics relative to the fundamental periods of the fixed-base system and those of the respective SSFS system. Increase in the response occurs when the period of the SSFS system is located on an ascending branch of the spectrum.

3. Based on the median structural responses, detrimental effects of SFSI are more pronounced for nonlinear structures; this implies that the evaluation of SFSI effects based on linear systems is unconservative.

\section{REFRENCES}

Applied Technology Council 1984. Tentative Provisions for the Development of Seismic Regulations for Buildings; ATC-3-06: California.
Dutta, S.C. \& Bhattacharya, K. \& Roy, R. 2004. Response of low-rise buildings under seismic ground excitation incorporating soil-structure interaction. Soil Dynamics and Earthquake Engineering 24:893-914.

FEMA 440 2005. Recommended Improvements of Nonlinear Static Seismic Analysis Procedures. Applied Technology Council: California.

Gazetas, G. \& Mylonakis, G. 1998. Seismic soil-structure interaction: new evidence and emerging issues. Geotechnical Earthquake Engineering and Soil Dynamics 3: proceedings of speciality conference (ASCE): 1119-74.

Jennings, P.C. \& Beilak J. 1973. Dynamics of building-soil interaction. Bulletin of the Seismological Society of America 63(1):9-48.

Jin, S. \& Lutes, L.D. \& Sarkani S. 2000. Response variability for a structure with soil-structure interactions and uncertain soil properties. Probabilistic Engineering Mechanics 15: 175-183.

Lutes, L.D. \& Sarkani, S. \& Jin, S. 2000. Response variability of an SSI system with uncertain structural and soil properties. Engineering Structures 22:605-620.

Mylonakis, G. \& Gazetas, G.2000. Seismic soil-structure interaction: beneficial or detrimental. Journal of Earthquake Engineering 4(3): 227-301.

Shome, N. \& Cornell, C.A. \& Bazzurro, P. \& Carballo J.E. 1998. Earthquakes, records, and nonlinear responses. Earthquake Spectra 14(3): 469-500.

Stewart, J.P. \& Kim S. \& Bielak J. \& Dobry R. \& Power M.S. 2003. Revisions to soil-structure interaction procedures in NEHRP design provisions. Earthquake Spectra 19(3): 67796.

Veletsos, A.S. \& Meek, J.W. 1974. Dynamic behaviour of building-foundation systems. Earthquake Engineering and Structural Dynamics 3:121-38.

Veletsos, A.S. \& Nair V.D. 1975. Seismic interaction of structures on hysteresis foundations. Journal of the structural division (ASCE) 101:109-129.

Wolf, J.P. 1994. Foundation Vibration Analysis Using Simple Physical Model. Englewood Cliffs, NJ: Prentice-Hall. 